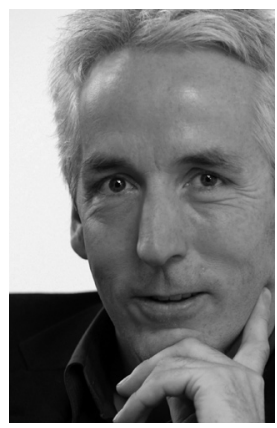

Prof. (FH) Dr. iur. Kurt Pärli, Dozent und Forschungsleiter am Institut für Wirtschaftsrecht der School of Management and Law, Züricher Hochschule für angewandte Wissenschaften
Wenn Grenzgänger/innen, die in der Schweiz gearbeitet haben und in Deutschland leben, arbeitsunfähig werden und Leistungen schweizerischer Sozial- oder Privatversicherungen beziehen, stellt sich die Frage, wie diese Leistungen nach deutschem Steuerrecht zu qualifizieren sind. Durch das Freizügigkeitsabkommen der Schweiz mit der EU und ihren Mitgliedstaaten sind solche Fragen auch im Lichte der Arbeitnehmerfreizügigkeit zu behandeln. Zu prüfen ist insbesondere, ob keine Diskriminierung der Grenzgänger/innen bei steuerlichen Vergünstigungen vorliegt.

\title{
Steuerliche Folgen schweizerischer Leistungen bei Arbeitsunfähigkeit für Grenzgänger aus Deutschland
}

\author{
Prof. Dr. Kurt Pärli ${ }^{1}$
}

\section{Einleitung}

Das Personenfreizügigkeitsabkommen der Schweiz mit der EU und den EU-Mitgliedstaaten (FZA) ${ }^{2}$ erleichtert Arbeitskräften aus dem EU-Raum den Zugang zum schweizerischen Arbeitsmarkt. Besonders Arbeitnehmer aus Deutschland machen von der Freizügigkeit Gebrauch und arbeiten für längere oder kür-

1 Der Autor dankt seinem Assistenten, Andreas Petrik, MA of Law, für die Mitwirkung an diesem Beitrag. Im Beitrag ist die Rechtsprechung bis zum 30. April 2008 berücksichtigt.

2 SR 0.142.112.681; ABl Nr. L 114 vom 30.4.2002, S. 6, geändert durch den Beschluss Nr. 2/2003 des Gemischten Ausschusses vom 15.7.2003, ABl Nr. L 187 vom 26.7.2003, S. 55.

zere Zeit in der Schweiz ${ }^{3}$. Die Schweiz hat im FZA die gemeinschaftsrechtlichen Bestimmungen zur Arbeitnehmerfreizügigkeit praktisch vollständig übernommen ${ }^{4}$. Für die EU-Mitglied-

\footnotetext{
3 Von 4.122.000 Arbeitnehmenden in der Schweiz waren im 2. Quartal 2007 115.000 aus Deutschland (ständige Wohnbevölkerung); siehe Schweizerische Arbeitskräfteerhebung, auf http://www.bfs.admin.ch/bfs/portal/de/index/themen/03/02/blank/data/03.Document.100730.xls (besucht am 30.4.2008).

4 Imhof, Schweizerische Leistungen bei Mutterschaft und FZW/Europarecht, in Gächter (Hg.), Das europäische Koordinationsrecht der sozialen Sicherheit und die Schweiz, 2006, S. 160 f.; Bergmann, Das europäische Sozialrecht des Freizügigkeitsabkommens EU-Schweiz, NZS 2002, 220; siehe auch EuGH v. 6. 6. 2006 - Rs. C-339/05, Zentralbetriebsrat der Landeskrankenhäuser Tirols ./. Land Tirol, Schlussanträge des Generalanwalts (Das Vorabentscheidungsgesuch wurde zurückgezogen), Rz. 40-41.
} 
staaten handelt es sich beim Freizügigkeitsabkommen um ein Assoziierungsabkommen im Sinne von Art. 310 EGV, das unmittelbare Wirkung entfaltet ${ }^{5}$. Im Anwendungsbereich des Abkommens sind grenzüberschreitende Sachverhalte (auch) im Lichte der Arbeitnehmerfreizügigkeit zu beurteilen ${ }^{6}$. Dies betrifft unter anderem die Anrechnung von im EU-Ausland erworbenen Dienstjahren ${ }^{7}$. Die Regelungen zur Arbeitnehmerfreizügigkeit verlangen auch volle Gleichbehandlung bei sozialen und steuerlichen Vergünstigungen (Art. 7 Abs. 2 V0 1612/68 EWG ${ }^{8}$ ). Auch Grenzgängern und Grenzgängerinnen sind die gleichen steuerlichen Vergünstigungen zu gewähren wie Arbeitnehmern, die im gleichen Staat wohnen und arbeiten $^{9}$.

Die Steuer- und Sozialsysteme der EU-Mitgliedstaaten sind unterschiedlich, entsprechend schwierig gestaltet sich die steuerliche Behandlung grenzüberschreitender Sozialleistungen $^{10}$. In diesem Beitrag werden die Steuerfolgen für in Deutschland lebende Grenzgängerinnen und Grenzgänger erörtert, die schweizerische Sozialleistungen wegen Arbeitsunfähigkeit beziehen bzw. bezogen haben. Nach § 3 Ziff. 1 lit. 9 des deutschen Einkommensteuergesetzes (EStG) ${ }^{11}$ werden Leistungen aus Kranken- und Unfallversicherungen als steuerfreie Versicherungsleistungen behandelt. Solche Versicherungsleistungen liegen auch dann vor, wenn der Versicherungsschutz im Zusammenhang mit einem Arbeitsverhältnis gewährt wird. Demgegenüber werden vom Arbeitgeber geleistete Entgeltfortzahlungen im Krankheitsfall grundsätzlich als zu versteuernder Arbeitslohn qualifiziert ${ }^{12}$.

Die schweizerische Rechtslage bezüglich der Entgeltfortzahlungen des Arbeitgebers und Leistungen von Krankentaggeldversicherungen, der Mutterschaftsversicherung sowie der Unfallversicherung weist einige Besonderheiten auf und lässt sich nicht ohne weiteres mit der deutschen Rechtslage vergleichen. Die richtige steuerrechtliche Erfassung nach deutschem Steuerrecht setzt deshalb genaue Kenntnis der schweizerischen Regelungen voraus. Darüber hinaus gilt es zu beach-

5 EuGH v. 6.6.2006 - Rs. C-339/05, Zentralbetriebsrat der Landeskrankenhäuser Tirols ./. Land Tirol, Schlussanträge des Generalanwalts (Das Vorabentscheidungsgesuch wurde zurückgezogen), Rz. 39.

6 Pärli, Arbeitsrechtliches Diskriminierungsverbot europäischer Wanderarbeitnehmer nach Gemeinschaftsrecht und nach dem Personenfreizügigkeitsrecht mit der Schweiz, ZESAR 2007, S. $33 \mathrm{f}$.

7 EuGH v. 30.9.2003 - Rs. C-224/01, Gerhard Köbler ./. Republik Österreich, Rz. 73 f.; EuGH v. 12.3. 1998 - Rs. C-187/96, Kommission ./. Griechenland, Rz. 20 f.; EuGH v. 16. 6. 1987 - Rs. 225/85, Kommission ./. Italien, Rz. 14; EuGH v. 15. 1. 1998 - Rs. C-15/96, Schöning-Kongebetopoulo ./. Freie und Hansestadt Hamburg, Rz. 28.

8 Verordnung (EWG) Nr. 1612/68 des Rates vom 15. 10. 1968 über die Freizügigkeit der Arbeitnehmer innerhalb der Gemeinschaft, ABl. Nr. L 257 vom 19. 10. 1968, S. 2.

9 Brechmann, N 65 zu Art. 39 EGV, in Callies/Ruffert, Kommentar zu EU-Vertrag und EG-Vertrag, 3. Auflage, 2007; EuGH v. 18.7.2007 - Rs. C-212/05, Hartmann, Rz. 20.

10 Siehe dazu: Rentzsch, Systeme sozialer Sicherheit und Steuerrecht in Europa - Kollision durch Koordination? Freizügigkeitsabkommen im Internationalen Steuerrecht der Schweiz, Zeitschrift für Schweizerisches und Internationales Steuerrecht, ZSIS 2007, Aufsätze Nr. 1, S. 1 ff.

11 Einkommensteuergesetz in der Fassung der Bekanntmachung vom 19. 10. 2002 (BGBl. I S. 4210; 2003 I S. 179).

12 Siehe dazu hinten, III. Steuerfolgen für GrenzgängerInnen in Deutschland. ten, dass grenzüberschreitende Sachverhalte vom Anwendungsbereich des Personenfreizügigkeitsabkommens erfasst sind. Wer in Deutschland lebt und in der Schweiz arbeitet oder gearbeitet hat, fällt unter den Anwendungsbereich dieses Abkommens ${ }^{13}$ und hat grundsätzlich Anspruch auf gleiche steuerliche und soziale Vergünstigungen wie inländisch Beschäftigte, die keinen grenzüberschreitenden Sachverhalt verwirklicht haben.

Nachfolgend wird ausführlich dargestellt, welche finanziellen Ansprüche Arbeitnehmern nach schweizerischem Recht bei krankheits- oder unfallbedingter Arbeitsunfähigkeit und bei Mutterschaft zustehen.

Anschließend werden die Steuerfolgen der schweizerischen Leistungen bei Arbeitsunfähigkeit in Deutschland behandelt und im letzten Teil des Beitrages folgen einige Bemerkungen zur Bedeutung des Personenfreizügigkeitsabkommens im Hinblick auf die Besteuerung von Einkommen bei grenzüberschreitenden Konstellationen.

\section{Leistungen bei Krankheit, Unfall, Mutterschaft}

\section{Die gesetzliche Ausgestaltung bei öffentlichrechtlichen Beschäftigungen}

Die Eidgenossenschaft (der Bund), die Kantone (die Länder) und die Gemeinden (die Kommunen) sowie andere öffentlichrechtliche Körperschaften sind hinsichtlich der Ausgestaltung der Arbeitsbedingungen nicht den Bestimmungen im schweizerischen Obligationenrecht (OR) ${ }^{14}$ zum Arbeitsvertrag (Art. 319 bis 362 OR) unterworfen. Das Arbeitsverhältnis im öffentlichrechtlichen Bereich wird vielmehr durch das jeweils anwendbare öffentlichrechtliche Personalrecht bestimmt ${ }^{15}$. Soweit das öffentliche Personalrecht Lücken aufweist, werden die einschlägigen OR-Bestimmungen analog angewendet ${ }^{16}$. Im Bereich des Bundespersonalrechts ist dieses Vorgehen in Art. 6 Abs. 2 des Bundespersonalgesetzes (BPG) ${ }^{17}$ ausdrücklich vorgesehen.

Das jeweils anwendbare öffentliche Personalrecht bestimmt die Voraussetzungen und die Dauer der Entgeltfortzahlungsleistungen bei krankheits- oder unfallbedingter Arbeits-

\footnotetext{
13 Siehe dazu die Schlussanträge des Generalanwaltes in der Rechtssache EuGH v. 6.6. 2006 - Rs. C339/05, Zentralbetriebsrat der Landeskrankenhäuser Tirols ./. Land Tirol (Das Vorabentscheidungsgesuch wurde zurückgezogen). In der Sache ging es um die Frage, ob Beschäftigungszeiten, die vor Inkrafttreten des Abkommens in der Schweiz zurückgelegt wurden, für die Bestimmungen des Dienstalters bei einer gegenwärtig in Österreich ausgeübten Tätigkeit anzurechnen sind. Der Generalanwalt bejahte dies und befürwortete insbesondere auch eine unmittelbare Wirkung des Personenfreizügigkeitsabkommens. Im Ergebnis konnten sich die österreichischen Arbeitnehmerinnen gegenüber Österreich auf das Personenfreizügigkeitsabkommen mit der Schweiz beziehen.

14 Bundesgesetz vom 30.3. 1911 betreffend die Ergänzung des Schweizerischen Zivilgesetzbuches (Fünfter Teil: Obligationenrecht) (SR 220).

15 Portmann in Basler Kommentar, Honsell / Vogt/ Wiegand (Hg.), 4. Auflage, 2007, N 1 zu Art. 342 OR.

16 Siehe dazu grundlegend: BGE 124 II 53 E. 2. Die Lückenfüllung durch Bestimmungen betrifft nicht nur die Art. 319 bis 362 zum Arbeitsvertrag, auch die Bestimmungen zum Allgemeinen Teil des OR können herangezogen werden, siehe 132 II 161, E. 3.

17 Bundespersonalgesetz vom 24.3.2000 (SR 172.220.1).
} 
unfähigkeit. Eine Darstellung sämtlicher kantonaler, kommunaler und anderer öffentlichrechtlichen Bestimmungen zur Entgeltfortzahlung würde den Rahmen dieses Beitrages bei weitem sprengen. Es wird deshalb nur die Entgeltfortzahlung nach Bundespersonalgesetz (PBG) erläutert. Gestützt auf Art. 29 BPG regelt die Rahmenverordnung des Bundesrates (BPV) ${ }^{18}$ den Lohnanspruch bei Krankheit und Unfall in Art. 56 BPV wie folgt: Der Arbeitgeber bezahlt den vollen Lohn während zwölf Monaten (Art. 56 Abs. 1 BPV) und nach Ablauf dieser Frist 90 Prozent des Lohnes während weiterer zwölf Monate. Dabei darf die Summe des gekürzten Lohnes nicht geringer sein als die Leistungen der obligatorischen Unfallversicherung oder als die Leistungen der Pensionskasse Publica ${ }^{19}$, auf die der Angestellte bei Invalidität Anspruch hätte (Art. 56 Abs. 2 BPV). Nach Ablauf von insgesamt 24 Monaten Lohnfortzahlung kann gemäß Art. 56 Abs. 3 BPV in begründeten Ausnahmefällen ein weiterer Anspruch von zwölf Monaten Lohnfortzahlung im Umfang von 90 Prozent bestehen. Ein solcher Anspruch erlischt innerhalb der Zusatzfrist von zwölf Monaten auf den Zeitpunkt des Abschlusses medizinischer Abklärungen oder wenn eine Rente ausgerichtet wird. Nach insgesamt 36 Monaten erlischt der Lohnfortzahlungsanspruch unabhängig vom Weiterbestehen eines Arbeitsverhältnisses.

Die Gehaltsfortzahlung nach Bundespersonalrecht geht im Vergleich zu anderen öffentlichrechtlichen Regelungen und erst recht im Vergleich zur Minimalregelung nach Obligationenrecht sehr weit ${ }^{20}$.

\section{Die gesetzliche Ausgestaltung bei privatrechtlichen Anstellungsverhältnissen}

\section{a) Die Regelung in Art. 324a Abs. 1-3 OR}

Der Einzelarbeitsvertrag ist in den Art. 319 bis 362 OR geregelt. Diese Bestimmungen sind zum Teil zwingender (absolut oder relativ) und zum Teil dispositiver Natur. Dispositive Normen dürfen von den Vertragsparteien abgeändert werden. Von relativ zwingenden Bestimmungen darf nur zu Gunsten der Arbeitnehmenden abgewichen werden, während die absolut zwingenden Normen jegliche Abweichung verbieten ${ }^{21}$. Die Bestimmung zur Entgeltfortzahlung in Art. 324a OR ist relativ zwingend, d.h. es darf nur zu Gunsten der Arbeitnehmenden abgewichen werden.

Nach Art. 324a Abs. 1 OR hat der Arbeitgeber dem Arbeitnehmer unter bestimmten Voraussetzungen den Lohn trotz ausfallender Arbeit für eine beschränkte Zeit weiter aus-

\footnotetext{
18 Bundespersonalverordnung vom 3.7.2001 (SR 172.220.111.3).
}

19 Publica, die Pensionskasse des Bundes, ist eine öffentlich-rechtliche Anlage des Bundes mit eigener Rechtspersönlichkeit; weiter Informationen unter www.publica.ch; Bundesgesetz vom 23.6.2000 über die Pensionskasse des Bundes (SR 172.222.0).

20 Portmann, Zum Inkrafttreten des Bundespersonalgesetzes und der Bundespersonalverordnung, Darstellung einiger Schwerpunkte mit Hinweisen zur obligationenrechtlichen Regelung des Arbeitsvertrages, Zeitschrift für Arbeitsrecht und Arbeitslosenversicherung, 2001, S. 182.

21 Art. $361 \mathrm{f}$. OR; siehe hierzu: Streiff/v. Kaenel, Arbeitsvertrag, 6. Auflage, 2005, Art. 361 f.; Portmann in Basler Kommentar, 4. Auflage, 2007, Art. 361 f. zurichten. Das Gesetz bezeichnet in einer nicht abschließenden $^{22}$ Liste die Gründe, die zu einer Lohnfortzahlungspflicht des Arbeitgebers führen: Krankheit, Unfall, Erfüllung gesetzlicher Pflichten und Ausübung eines öffentliches Amtes. Die dargelegten Gründe einer Lohnfortzahlungspflicht dürfen von den Arbeitnehmern nicht verschuldet verursacht worden $\operatorname{sein}^{23}$.

Im ersten Dienstjahr beträgt die Lohnfortzahlungspflicht nach Art. 324a Abs. 2 OR drei Wochen. Anschließend besteht der Lohnanspruch gemäß Wortlaut der Bestimmung für einen angemessenen Zeitraum. Was einen angemessenen Zeitraum darstellt, wird in der Praxis durch die so genannte Berner-, Basler- und Zürcherskala konkretisiert. Diese Skalen gehen vom Grundsatz aus, je länger das Dienstverhältnis gedauert hat, desto länger besteht ein Anspruch auf Lohnfortzahlung. Bei allen drei Skalen besteht erst nach mehr als 20 Dienstjahren ein sechsmonatiger Lohnfortzahlungsanspruch ${ }^{24}$.

Der dritte Absatz von Art. 324a OR sieht vor, dass der Arbeitgeber bei Schwangerschaft den Lohn im gleichen Umfang zu entrichten hat ${ }^{25}$. Eine Schwangerschaft als solche führt jedoch nicht zu einem Anspruch auf Lohn ohne Arbeitsleistung $^{26}$. Schwangere Arbeitnehmerinnen haben Anspruch auf die Lohnfortzahlung während einer beschränkten Zeit und soweit sie schwangerschaftsbedingt der Arbeit fernbleiben müssen. $\mathrm{Zu}$ beachten sind hier zudem Vorschriften zum Schutze der schwangeren Mitarbeiterin nach Arbeitsgesetz (ArG) ${ }^{27}$. Nach Art. 35 Abs. 2 ArG und Art. 62 ff. der Verordnung 1 zum ArG (ArGV 1) ${ }^{28}$ dürfen schwangere und stillende Frauen keine schwere und gefährliche Arbeit ausführen. Soweit der Arbeitgeber keine Ersatzarbeit anbieten kann, muss er der schwangeren Arbeitnehmerin 80 Prozent des Lohnes weiterhin ausrichten $^{29}$. Diese Lohnfortzahlung stützt sich auf zwingendes öffentliches Recht und wird nicht auf den Lohnfortzahlungsanspruch nach Art. 324a Abs. 1 und 2 OR angerechnet. Anders verhält es sich, wenn die schwangere Arbeitnehmerin wegen Schwangerschaftsbeschwerden an der Arbeit verhindert ist. Nach umstrittener Lehre und Rechtsprechung verschafft Art. 324a Abs. 3 OR keinen eigenständigen Anspruch auf eine

22 In Frage kommen weitere Gründe wie z. B. die unverschuldete Untersuchungs haft, siehe dazu Brühwiler, Kommentar zum Einzelarbeitsvertrag, 2. Auflage, 1996, N 2 zu Art. 324a OR.

23 Portmann in Basler Kommentar, Honsell / Vogt/Wiegand (Hg.), 4. Auflage, 2007, N 4 - N 6 zu Art. 324a OR; Streiff/v. Kaenel, Arbeitsvertrag, 6. Auflage, 2005, N 29 zu Art. 324a/b OR.

24 Für eine Darstellung der verschiedenen Skalen siehe: Böhringer in Schweizer Vertrags-Handbuch, Münch/Böhringer/Kaspar/Propst (Hg.), 2007, S. $474 \mathrm{f}$.

25 Bis zum Inkrafttreten der obligatorischen Mutterschaftsversicherung (siehe dazu weiter hinten, II. 2. d) Mutterschaftsurlaub, Mutterschaftsentschädigung nach EOG und Leistungen des Arbeitgebers, S. 10) lautet der dritte Absatz von Art. 324a OR wie folgt: Bei Schwangerschaft und Niederkunft der Arbeitnehmerin hat der Arbeitgeber den Lohn im gleichen Umfang zu entrichten.

26 BGE 118 II 61, E. 2b. (= Pra 82 Nr. 142).

27 Bundesgesetz über die Arbeit in Industrie, Gewerbe und Handel vom 13.3. 1964 (SR 822.11).

28 Verordnung 1 zum Arbeitsgesetz vom 10.5.2000 (SR 822.111).

29 Wyler in Stämpflis Handkommentar zum Arbeitsgesetz, Geiser/v. Kaenel/ Wyler (Hg.), 2005, N 24 zu Art. 35 ArG. 
Lohnfortzahlung für eine beschränkte Zeit. Die durch schwangerschaftsbedingte Arbeitsunfähigkeit verursachten Abwesenheiten werden vielmehr an den „Kredit“ nach Art. 324a Abs. 2 OR angerechnet ${ }^{30}$. Bei dieser Rechtslage fragt sich, weshalb der Gesetzgeber überhaupt die Schwangerschaft in einem eigenständigen Absatz der Bestimmung zur Lohnfortzahlung geregelt hat. Die Erklärung besteht darin, dass der Gesetzgeber Ende der Sechziger Jahre klarstellen wollte, dass Arbeitsunfähigkeit wegen Schwangerschaft und Mutterschaft nicht als verschuldet anzusehen wären und demzufolge kein Anspruch auf Lohnfortzahlung bestanden hätte ${ }^{31}$.

\section{b) Abweichende Regelungen gemäss Art. 324a Abs. 4 OR}

Art. 324a Abs. 4 OR sieht vor, dass durch schriftliche Abrede, Normalarbeitsvertrag oder Gesamtarbeitsvertrag (Deutschland: Tarifvertrag) eine von Art. 324 Abs. 1-3 OR abweichende Regel getroffen werden kann, wenn diese für den Arbeitnehmer mindestens gleichwertig ist. Eine Abänderung zu Ungunsten der Arbeitnehmenden ist nicht zulässig; eine gleichwertige Regelung muss nach Gesetztext schriftlich vereinbart werden. $\mathrm{Zu}$ unterscheiden sind gleichwertige und für die Arbeitnehmenden bessere Lösungen; zu Gunsten der Arbeitnehmenden kann die Abweichung auch konkludent erfolgen ${ }^{32}$.

Das Gesetz schreibt nicht vor, auf welche Weise die Gleichwertigkeit der Lohnersatzzahlungsleistung zu erfolgen hat und es fehlen Anhaltspunkte, wann Gleichwertigkeit der Regelung gegeben ist. Häufig schließt der Arbeitgeber mit einem Versicherer eine Kollektiv-Krankentaggeldversicherung $\mathrm{ab}^{33}$. Diese Versicherungen sehen i.d.R. die Ausrichtung von Versicherungsleistungen für die Dauer von 720 Tagen vor. Die Höhe der Versicherungsleistung beträgt entweder 80 oder 100 Prozent des Gehaltes. Bezüglich Prämientragung (Arbeitgeber oder Arbeitnehmer oder Prämienteilung) ist die Praxis ebenfalls vielfältig, d.h., je nach Betrieb oder Branche kommen unterschiedliche Lösungen vor ${ }^{34}$. Damit die in Art. 324a OR vorgeschriebene Gleichwertigkeit erreicht ist, darf keine Lösung vorgesehen sein, die ausschließlich die Arbeitnehmer zur Übernahme der Prämie verpflichtet. Die Gleichwertigkeit der Versicherungsleistungen im Vergleich zur gesetzlichen Minimallö-

30 Brühwiler, Kommentar zum Einzelarbeitsvertrag, 2. Auflage, 1996, N 10c zu Art. 324a OR; Streiff/v. Kaenel, Arbeitsvertrag, 6. Auflage, 2005, N 25 zu Art. 324a/b OR; BGE v. 18. 12. 1992 (JAR 1993, S. 143), BGE

v. 17.11.1994 (JAR 1995, S. 112) und BGE v. 6. 4. 1995 (JAR 1995, S. 128); seco Merkblatt über den Schutz der Arbeitnehmerinnen bei Mutterschaft, 2001, S.9; anderer Ansicht: Egli in AJP 2000, S. 1074, Lohnfortzahlung und Versicherungsschutz gemäss Art. 324a OR; Vischer, Der Arbeitsvertrag, 3. Auflage, 2006, S. 132

31 Streiff/v. Kaenel, Arbeitsvertrag, 6. Auflage, 2005, N 25 zu Art. 324a/b OR; Portmann in Basler Kommentar, Honsell / Vogt/Wiegand (Hg.), 4. Auflage, 2007, N 31 zu Art. 324a OR.

32 BGE 131 III 634, E. 2.5.1. (= Pra 95 Nr. 131).

33 Siehe dazu weiter hinten, II. 3. Leistungen durch die Kollektiv-Krankentaggeldversicherung, S. 12.

34 Streiff/v. Kaenel, Arbeitsvertrag, 6. Auflage, 2005, N 23 zu Art. 324a/b; siehe dazu auch weiter hinten, II. 3.3. Leistungen durch die Kollektiv-Krankentaggeldversicherung c) Leistungen und Finanzierung, S. 12; zur Unterscheidung von Krankentaggeldversicherungen nach KVG und VVG vgl.: Eugster, Vergleich der Krankentaggeldversicherung (KTGV) nach KVG und nach VVG, in: Krankentaggeldversicherung: Arbeits- und versicherungsrechtliche Aspekte, in v. Kaenel (Hg.), 2007, S. $47 \mathrm{ff}$. sung nach Art. 324a Abs. 1-3 OR ist nach einer abstrakten Gesamtbetrachtung vorzunehmen ${ }^{35}$. Damit ist auch eine Lösung noch gleichwertig, die den Beginn der Lohnzahlungen bei Krankheit erst nach einer Karenzzeit von wenigen Tagen erbringt, obwohl eine solche Karenzzeit in Art. 324a Abs. 1-3 OR nicht vorgesehen ist. Voraussetzung ist indes, dass dieser für die Arbeitnehmenden nachteiligen Regelung gegenüber den minimalen gesetzlichen Vorschriften Vorteile, z.B. hinsichtlich Dauer der Lohnfortzahlung, gegenüberstehen ${ }^{36}$.

\section{c) Ausnahmen von der Lohnfortzahlungspflicht nach Art. 324b OR}

Ein Lohnfortzahlungsanspruch nach Art. 324a OR wird durch Art. 324b OR in den Fällen ausgeschlossen, in denen die Arbeitnehmenden auf Grund gesetzlicher Vorschriften gegen die wirtschaftlichen Folgen unverschuldeter Arbeitsverhinderung aus Gründen, die in seiner Person liegen, obligatorisch versichert ist und der Versicherungsanspruch mindestens 80 Prozent des ausfallenden Lohnes beträgt. Ist die Versicherungsleistung geringer, so hat der Arbeitgeber die Differenz zwischen der effektiven Versicherungsleistung und 80 Prozent des Lohnes zu entrichten. Diese Ergänzungszahlungspflicht trifft den Arbeitgeber nur dann, wenn die Voraussetzungen von Art. 324a Abs. 1-3 OR erfüllt sind. Auch für die Dauer der Lohnergänzungspflicht gilt der Zeitrahmen gemäß Art. 324a Abs. $2 \mathrm{OR}^{37}$.

Art. 324b OR bildet eine Koordinationsregel zwischen Arbeits- und Sozialversicherungsrecht ${ }^{38}$. Bei den in Art. 324b OR erwähnten obligatorischen Versicherungen handelt es sich um: die Militärversicherung aufgrund des Militärversicherungsgesetzes (MVG) ${ }^{39}$, die Unfallversicherung nach UVG, die Invalidenversicherung ${ }^{40}$ bezüglich der Leistung von Taggeldern und Renten sowie die Versicherung für Ersatzerwerb bei Militär-, Zivil- und Schutzdienst (Ersatzerwerbsordnung, EO) ${ }^{41}$. Umstritten ist, ob es sich auch bei der Mutterschaftsversicherung bzw. den Mutterschaftsleistungen nach dem Ersatzerwerbsordnungsgesetz EOG um eine obligatorische Versicherung im Sinne von Art. 324b OR handelt ${ }^{42}$.

\section{BGE 128 II 13, E. 5c/bb (= Pra 61 Nr. 172).}

36 Streiff/v. Kaenel, Arbeitsvertrag, 6. Auflage, 2005, N 24 zu Art. 324a/b OR; Portmann in Basler Kommentar, Honsell/Vogt/Wiegand (Hg.), 4. Auflage, 2007, N 51 zu Art. 324a OR; Schönenberger/Staehelin in Zürcher Kommentar, Staehelin/Vischer (Hg.), 3. Auflage, 1996, N 64 zu Art. 324a OR; Vischer, Der Arbeitsvertrag, 3. Auflage, 2006, S. 133.

37 Staehelin in Zürcher Kommentar, Staehelin/Vischer (Hg.), 3. Auflage, 1996, N 13 f. zu Art. 324b OR; Portmann in Basler Kommentar, Honsell/Vogt/Wiegand (Hg.), 4. Auflage, 2007, N 1 zu Art. 324b OR.

38 Brühwiler, Kommentar zum Einzelarbeitsvertrag, 2. Auflage, 1996, Vorbemerkung zu Art. 324b OR.

39 Bundesgesetz vom 19.6. 1992 über die Militärversicherung (SR 833.1).

40 Bundesgesetz vom 19.6. 1959 über die Invalidenversicherung (SR 831.20); die Qualifizierung von IV-Leistungen als eine obligatorische Leistung nach Art. 324b OR ist in der Lehre teilweise umstritten, siehe die Nachweise bei Streiff / v. Kaenel, Arbeitsvertrag, 6. Auflage, 2005, N 31 zu Art. 324a/b OR.

41 Bundesgesetz über den Erwerbsersatz für Dienstleistende und bei Mutterschaft (SR 834.1).

42 Siehe dazu hinten, II. 2. d) Mutterschaftsurlaub, Mutterschaftsentschädigung nach EOG und Leistungen des Arbeitgebers. 


\section{d) Mutterschaftsurlaub, Mutterschaftsentschädigung nach EOG und Leistungen des Arbeitgebers}

Nach Art. 329f OR hat die Arbeitnehmerin nach der Niederkunft Anspruch auf einen Mutterschaftsurlaub von mindestens 14 Wochen. Diese Bestimmung wurde gleichzeitig mit der Einführung der obligatorischen Mutterschaftsversicherung am 1. Juli 2005 eingeführt. Die Bestimmung gewährt der Arbeitnehmerin nicht einen Lohnanspruch, vielmehr besteht die Rechtsfolge von Art. 329f OR in einem Anspruch auf das Ruhen der Arbeitspflicht während der genannten Zeitdauer von 14 Wochen. Das Arbeitsverhältnis dauert weiter, auch wenn während den 14 Wochen die Hauptleistungspflicht der Arbeitgeberin, die Lohnzahlung, mangels anderer vertraglicher Vereinbarung ${ }^{43}$, wie die Arbeitspflicht der Arbeitnehmerin ebenfalls ruht.

Während des Mutterschaftsurlaubes hat die Arbeitnehmerin nach Art. 16e EOG einen Anspruch auf eine als Taggeld ausgerichtete Mutterschaftsentschädigung von 80 Prozent des durchschnittlichen Erwerbseinkommens vor Beginn des Anspruches. Der Taggeldanspruch ist beschränkt auf maximal 172 Franken pro Tag (Stand 1.1.2008) ${ }^{44}$. Nach Art. 16b EOG haben die Frauen Anspruch auf Mutterschaftsentschädigung, die zum Zeitpunkt der Geburt ihres Kindes entweder Arbeitnehmerinnen oder Selbstständige ${ }^{45}$ sind, oder im Betrieb des Ehemannes mitarbeiten und einen Barlohn beziehen, oder arbeitslos sind und ein Taggeld der Arbeitslosenversicherung beziehen oder die Anspruchsvoraussetzungen für Taggelder der Arbeitslosenversicherung erfüllen würden, oder wegen Krankheit, Unfall oder Invalidität arbeitsunfähig sind und Taggeldleistungen einer Sozial- oder Privatversicherung beziehen, oder in einem gültigen Arbeitsverhältnis stehen, aber keine Lohnfortzahlung oder Taggeldleistung erhalten, weil der Anspruch ausgeschöpft ist ${ }^{46}$.

Wer eine Mutterschaftsentschädigung geltend machen will, muss während neun Monaten vor der Geburt im Sinne des Bundesgesetzes über die Alters-, Hinterbliebenen- und Invalidenversicherung (AHVG) obligatorisch versichert gewesen sein. In dieser Zeitspanne muss zudem während fünf Monaten eine sozialversicherungspflichtige Erwerbstätigkeit ausgeübt worden sein. In Übereinstimmung mit dem Diskriminierungsverbot des Personenfreizügigkeitsabkommen werden gestützt auf Art. 28a EOG und Art. $28 \mathrm{EOV}^{47}$ Versicherungs- und Beschäftigungszeiten in EU- und EFTA-Staaten angerechnet ${ }^{48}$. Der An-

43 So auch Streiff/v. Kaenel, Arbeitsvertrag, 6. Auflage, 2005, N 8 zu Art. 329f OR

44 Art. $16 \mathrm{f}$ EOG.

45 BGE 133 V 73 E.4 (= Pra 97 Nr. 10): bei gesetzmässiger Auslegung von Art. 30 Abs. 1 lit. a EOV hat eine Selbstständigerwerbende, die im Zeitpunkt der Niederkunft vorübergehend arbeitsunfähig war, auch dann Anspruch auf Mutterschaftsentschädigung, wenn sie nicht über ein Ersatzeinkommen verfügt.

46 Siehe zum Ganzen: Bruchez, La nouvelle aussurance-maternité et ses effets sur le droit du contrat de travail, in: La Semaine Judiciaire, 2005 I, S. 247 ; Subilia, La nouvelle loi sur les allocations pour pertre de gain et maternité, in: AJP, 2005, S. $1472 \mathrm{f}$.

47 Verordnung zum Erwerbsersatzgesetz (SR 834.11).

48 Botschaft des Bundesrates zur parlamentarischen Initiative betreffend Ausweitung der Erwerbsersatzansprüche auf erwerbstätige Mütter (BBl. 2003 1119). spruch auf die Mutterschaftsentschädigung beginnt mit der Niederkunft und endet nach vierzehn Wochen (Art. 16c Abs. 1 EOG) und kann von der Mutter selbst, ihrem Arbeitgeber oder Angehörigen (wenn die Mutter ihren Unterhalts- oder Unterstützungspflichten nicht nachkommt) geltend gemacht werden.

\section{e) Die Rechtslage bei unfallbedingter Arbeitsunfähigkeit}

Nach Art. 1 des Bundesgesetzes über die obligatorische Unfallversicherung (UVG ${ }^{49}$ sind alle in der Schweiz beschäftigten Arbeitnehmer und Arbeitnehmerinnen obligatorisch unfallversichert. Die Unfallversicherung deckt die Risiken von Berufsunfällen, Nichtberufsunfällen und Berufskrankheiten (Art. 6 UVG) ab. Teilzeitbeschäftigte mit einer wöchentlichen Arbeitszeit von weniger als acht Stunden sind für Nichtberufsunfälle nicht nach UVG unfallversichert (Art. 7 Abs. 2 und Art. 8 Abs. 2 UVG in Verbindung mit Art. 13 UVV). Die Versicherten haben unter anderem Anspruch auf Taggelder, Renten, Integritätsentschädigungen und Abfindungen. Taggelder und Renten werden nach dem versicherten Verdienst bemessen (Art. 15 Abs. 1 UVG). Als versicherter Verdienst gilt für die Bemessung der Taggelder der letzte vor dem Unfall bezogene Lohn. Der Anspruch auf das Taggeld in der Höhe von 80 Prozent des versicherten Verdienstes besteht solange, wie der Versicherte infolge des Unfalles ganz oder teilweise arbeitsunfähig ist (Art. 16 und 17 UVG).

Die Taggeldleistung der obligatorischen Versicherung tritt vollständig an die Stelle der Lohnfortzahlungspflicht des Arbeitgebers, sofern und soweit die Versicherungsleistungen mindestens 80 Prozent des Lohnes betragen ${ }^{50}$. Beträgt die Versicherungsleistung weniger als 80 Prozent, so muss die Arbeitgeberin die Differenz entrichten (Art. 324b Abs. 2 OR).

Werden die Versicherungsleistungen erst nach einer Wartezeit gewährt, so hat der Arbeitgeber für diese Zeit mindestens 80 Prozent des Lohnes zu entrichten (Art. 324b Abs. 3 OR). Die obligatorische Unfallversicherung richtet nach Art. 16 Abs. 2 UVG ein Taggeld von 80 Prozent des versicherten Lohnes am dritten Tage der unfallbedingten Arbeitsunfähigkeit aus. Dies hat zur Folge, dass die Arbeitgeberin an den beiden ersten Tagen eine Lohnfortzahlung (80 Prozent) leisten muss.

\section{Leistungen durch die Kollektiv-Krankentaggeldversicherung}

\section{a) Allgemeines}

Wie in den vorangehenden Ausführungen deutlich wurde, ist der gesetzliche Minimalschutz bei krankheitsbedingter Arbeitsunfähigkeit bescheiden. Anders als bei unfallbedingter Arbeitsunfähigkeit fehlt bei krankheitsbedingter Arbeitsunfähigkeit eine obligatorische Taggeldversicherung ${ }^{51}$. Anders verhält es

49 Bundesgesetz über die Unfallversicherung vom 20.3. 1981 (SR 832.20).

50 Streiff/v. Kaenel, Arbeitsvertrag, 6. Auflage, 2005, N 31 zu Art. 324a/b OR.

51 Gnaegi, Le droit du travailleur au salaire en cas de maladie, Diss., Neuenburg 1995, S. 135; Pärli, Datenaustausch zwischen Arbeitgeber und Versicherung, 2003, S. $36 \mathrm{f}$. 
sich bei der Krankenpflegeversicherung, diese ist für die gesamte Wohnbevölkerung obligatorisch ${ }^{52}$. Da bis zur allfälligen Ausrichtung von Erwerbsunfähigkeitsleistungen der Invalidenversicherung Wartezeiten bestehen, kann eine längerdauernde krankheitsbedingte Arbeitsunfähigkeit in Beschäftigungsverhältnissen ohne zusätzlichen Versicherungsschutz zu erheblichen existenziellen Problemen führen ${ }^{53}$. Politische Vorstöße für ein alle Arbeitsverhältnisse umfassendes Krankentaggeldobligatorium sind gescheitert ${ }^{54}$. Bei einer Mehrheit der Arbeitsverhältnisse wird indes der minimale gesetzliche Lohnfortzahlungsschutz durch Krankentaggeldversicherungen ergänzt ${ }^{55}$. Sehr oft finden sich in Gesamtarbeitsverträgen (Terminologie in Deutschland: Tarifverträge) Bestimmungen über die Verpflichtung des Arbeitgebers zum Abschluss einer Krankentaggeldversicherung ${ }^{56}$.

\section{b) Erscheinungsformen der Krankentaggeldversicherung}

Die Krankentaggeldversicherung kann als Einzel- oder als Kollektivversicherung abgeschlossen werden ${ }^{57}$. Mit einer Einzelversicherung versichert ein Arbeitnehmer oder eine Arbeitnehmerin auf eigene Initiative das Risiko länger dauernden Lohnausfalls. In der Einzelversicherung sind der Versicherungsnehmer und die versicherte Person identisch. In der Kollektivversicherung ist indes zwischen dem Versicherungsnehmer und der versicherten Person zu unterscheiden. Mit der Kollektivversicherung versichert eine Person als Versicherungsnehmer mehrere Personen, also Personengruppen ${ }^{58}$. Wer die Kollektivversicherung abschließt, ist Versicherungsnehmer, und wer durch sie Versicherungsschutz genießt, ist Versicher$\operatorname{ter}^{59}$.

Im vorliegenden Zusammenhang schließt der Arbeitgeber als Versicherungsnehmer mit einer Versicherung eine Kollektiv-Krankentaggeldversicherung mit dem Zweck ab, seine Lohnfortzahlungspflicht nach Art. 324a OR abzudecken und / oder für seine Arbeitnehmer/innen einen weitergehenden Schutz vorzusehen ${ }^{60}$. Möglich ist, dass der Arbeitgeber selbst, soweit es sich um eine natürliche Person handelt, die ihren ei-

52 Art. 3 KVG. Für Arbeitnehmende aus EU-Staaten siehe Art. 95a KVG in Verbindung mit Art. 1 Abs. 2 lit. d KVV.

53 Pärli / Müller/Spycher, Aids, Recht und Geld, 2003, S. 294 (Tabelle 97).

54 Pärli, Datenaustausch zwischen Arbeitgeber und Versicherung, 2003, S. 37.

55 Zur (unbefriedigten) Datenlage im Bereich der Kollektiv-Krankentaggeldversicherung siehe Pärli, Datenaustausch zwischen Arbeitgeber und Versicherung, 2003, S. $12 \mathrm{ff}$.

56 Fierz, Erscheinungsformen der KTGV in der Praxis, in v. Kaenel (Hg.), Krankentaggeldversicherung: Arbeits- und sozialversicherungsrechtliche Aspekte, 2007, S. 2; Müller, Grundlagen der Krankentaggeldversicherung nach VVG, in v. Kaenel (Hg.), Krankentaggeldversicherung: Arbeits- und sozialversicherungsrechtliche Aspekte, 2007, S. 23 f.

57 Eugster, Vergleich der Krankentaggeldversicherung (KTGV) nach KVG und nach VVG, in v. Kaenel (Hg.), Krankentaggeldversicherung: Arbeits- und sozialversicherungsrechtliche Aspekte, 2007, S. 48.

58 Maurer, Bundessozialversicherungsrecht, 1993, S. 271.

59 Maurer, Das neue Krankenversicherungsrecht, 1996, S. 109. Zur Unterscheidung zwischen Versicherungsnehmer und Versicherten siehe weiter Maurer, Privatversicherungsrecht, 3. Auflage, 1995.

60 Soweit der Versicherungsschutz über die nach Art. 324a Abs. 4 OR geforderte Gleichwertigkeit hinausgeht, ist dies eine im Sinne von Art. 362 OR zulässige Abweichung zu Gunsten der Arbeitnehmenden. genen Erwerbsausfall versichern will, gleichzeitig Versicherungsnehmer und Versicherter ist ${ }^{61}$. Die versicherten Arbeitnehmer/innen sind nicht Vertragspartei ${ }^{62}$. Es handelt sich hier vielmehr um einen so genannten echten Vertrag zu Gunsten Dritter im Sinne von Art. 112 OR $^{63}$.

Die Krankentaggeldversicherung kann nach dem sozialversicherungsrechtlichen Krankenversicherungsgesetz $(\mathrm{KVG})^{64}$ oder nach dem privatrechtlichen Versicherungsvertragsgesetz (VVG) ${ }^{65}$ abgeschlossen werden. Das KVG enthält in den Artikeln 67 bis 77 KVG Bestimmungen zum Beitritt ${ }^{66}$, den Versicherern $^{67}$, den Versicherungsvorbehalten ${ }^{68}$, den Leistungen $^{69}$, Freizügigkeitsrechten bei Austritt aus der Kollektivversicherung $^{70}$ und den Prämien ${ }^{71}$. Im Gegensatz zur obligatorischen Krankenpflegeversicherung nach KVG ist die freiwillige Taggeldversicherung nicht durchnormiert ${ }^{72}$. Die weitere Ausgestaltung der Versicherungsverhältnisse wird in den Allgemeinen Versicherungsbedingungen (AVB) und im Versicherungsvertrag geregelt. Ergänzend kommen die Bestimmungen des allgemeinen Teils des Obligationenrechts und das VVG zur Anwendung $^{73}$. Das VVG überlässt den Privatversicherern im Vergleich zum KVG mehr Spielraum für die Gestaltung der Versicherung, es enthält insbesondere weniger Sozialschutzbestimmungen ${ }^{74}$. In der Praxis werden deshalb wesentlich häufiger Kollektivkrankentaggeldversicherungen nach VVG abgeschlossen $^{75}$.

\section{c) Leistungen und Finanzierung}

Sowohl bei VVG- wie bei KVG-Kollektivtaggeldversicherungen vereinbaren die Versicherer mit dem Versicherungsnehmer Höhe und Dauer des versicherten Taggeldes. Im KVG-Bereich bestimmt Art. 72 Abs. 3 KVG, das Taggeld sei „für eine oder mehrere Erkrankungen während mindestens 720 Tagen inner-

\footnotetext{
61 Pärli, Datenaustausch zwischen Arbeitgeber und Versicherung, 2003, S. 41.
}

62 Müller, Grundlagen der Krankentaggeldversicherung nach VVG, in v. Kaenel (Hg.), Krankentaggeldversicherung: Arbeits- und sozialversicherungsrechtliche Aspekte, 2007, S. 30.

63 Weber, Kollektivversicherung bei anerkannten Krankenkassen, SZS 1968, S. 190. Siehe auch Bundesgerichtsentscheid (unveröffentlicht) v. 10. 12.1996, E. 3 b, K 78/96. Siehe zum direkten Forderungsrecht weiter hinten, II. 3. d.d) Direktes Forderungsrecht.

64 Bundesgesetz über die Krankenversicherung vom 18.3.1994 (SR 832.10). 65 Bundesgesetz über den Versicherungsvertrag vom 2.4. 1908 (SR 221.229.1).

66 Art. 67 KVG. Gemäss BGE 126 V 499, E. 2a handelt es sich beim KVG-Taggeldversicherungsvertrag zwischen der Versicherung und dem Versicherungsnehmer um einen verwaltungsrechtlichen Vertrag.

67 Art. 68 KVG.

68 Art. $69 \mathrm{KVG}$.

69 Art. $72-74$ KVG.

70 Art. $70-71 \mathrm{KVG}$.

71 Art. $76-77 \mathrm{KVG}$

72 Eugster, Die freiwillige Taggeldversicherung, in Meyer-Blaser, Soziale Sicherheit, 2. Auflage, 2007, N 1096.

73 Eugster, Die freiwillige Taggeldversicherung, in Meyer-Blaser, Soziale Sicherheit, 2. Auflage, 2007, N 1096; Pärli, Datenaustausch zwischen Arbeitgeber und Versicherung, 2003, S. $40 \mathrm{ff}$.

74 Eugster, Vergleich der Krankentaggeldversicherung (KTVG) nach KVG und VVG, in v. Kaenel (Hg.), Krankentaggeldversicherung: Arbeits- und versicherungsrechtliche Aspekte, 2007, S. 50 ff.

75 BGE v. 3.7.2001, 5C.41/2001 E. 2b/bb; Fierz, Krankentaggeldversicherung: Arbeits- und versicherungsrechtliche Aspekte, in v. Kaenel (Hg.), 2007, S. 2 ; Streiff/v. Kaenel, Arbeitsvertrag, 6. Auflage, 2005, N 13 zu Art. 324a/b OR. 
halb von 900 Tagen zu leisten“. Im KVG fehlt eine ausdrückliche Verankerung eines Mindesttaggeldes ${ }^{76}$. Bei VVG-KollektivKrankentaggeldversicherungen ist in der Regel vorgesehen, dass während 730 Tagen ein Taggeld in der Höhe von 80 bis $100 \%$ des versicherten Gehaltes ausgerichtet wird ${ }^{77}$. Maßgebend ist die vertragliche Vereinbarung zwischen der Versicherung und dem Arbeitgeber als Versicherungsnehmer. Oft sind die Modalitäten der Taggeldversicherung im Gesamtarbeitsvertrag (Deutschland: Tarifvertrag) vorgeschrieben ${ }^{78}$.

Bezüglich Prämiengestaltung ermöglicht Art. 77 KVG dem Versicherer, in der Kollektivversicherung von der Einzelversicherung abweichende Prämien festzulegen. Zur Prämientragung zwischen den versicherten Personen und dem Versicherungsnehmer findet sich im KVG keine Bestimmung. Prämienschuldner ist indes immer der Versicherungsnehmer, also vorliegend der Arbeitgeber. Wie weit sich die versicherten Arbeitnehmer an der Prämie zu beteiligen haben, ergibt sich aus dem Einzelarbeitsvertrag oder dem Gesamtarbeitsvertrag.

\section{d) Direktes Forderungsrecht}

Für die Qualifikation der schweizerischen Leistungen nach deutschem Steuerrecht ist maßgebend, ob die versicherten Arbeitnehmenden auf die Leistungen der Kollektivkrankentaggeldversicherung einen eigenen Anspruch haben ${ }^{79}$.

Nach Art. 87 VVG steht „demjenigen, zu dessen Gunsten die Versicherung abgeschlossen worden ist, mit dem Eintritt des Unfalls oder der Krankheit ein selbständiges Forderungsrecht gegen den Versicherer zu." Diese Bestimmung gilt nicht für die Kollektiv-Lebensversicherung. Hier sind die Versicherten nur dann anspruchsberechtigt, wenn sie im Vertrag als Begünstige bezeichnet werden (Art. 78 VVG) ${ }^{80}$. Die KollektivKrankentaggeldversicherung hingegen ist vom Anwendungsbereich des Art. 87 VVG erfasst ${ }^{81}$. In der Lehre und durch die Gerichte wurde das selbstständige Forderungsrecht auch damit begründet, dass im Kollektiv-Krankentaggeldversicherungsvertrag im Arbeitsverhältnis ein echter Vertrag zu Gunsten Dritter im Sinne von Art. 112 OR vorliege ${ }^{82}$. Art. 87 VVG ist indessen als lex specialis zum OR zu qualifizieren und das direkte Forderungsrecht ist deshalb auf Art. 87 VVG zu stützen ${ }^{83}$.

76 Largier / Schiavi, Krankentaggeld, Praxis rechtswidrig, plädoyer 2/1997, S. $17 \mathrm{f}$.

77 Fierz, Erscheinungsformen der KTGV-Versicherung in der Praxis, in v. Kaenel (Hg.), Krankentaggeldversicherung: Arbeits- und versicherungsrechtliche Aspekte, 2007, S. 9 f.

78 Müller, Grundlagen der Krankentaggeldversicherung nach VVG, in v. Kaenel (Hg.), Krankentaggeldversicherung: Arbeits- und sozialversicherungsrechtliche Aspekte, 2007, S. 23.

79 Siehe dazu hinten, III. Steuerfolgen für GrenzgängerInnen in Deutschland.

80 Weber, Privatversicherung, in Münch/Geiser (Hg.), Handbücher für die Anwaltspraxis, Band V, Schaden - Haftung - Versicherung, 1999, N 4.39.

81 BGE v. 10.11.2000, 120 V 38, E. 3b; BGE v. 10. 11. 2000, 4C.230/2000, E. 2a.

82 Weber, Kollektivversicherung bei anerkannten Krankenkasse, SZS 1968; S. 190; BGE 120 V 38, E. 3b. Siehe auch Bundesgerichtsentscheid (unveröffentlicht) v. 10.12.1996, E. 3 b, K 78/96.

83 Eugster, Vergleich der Krankentaggeldversicherung (KTVG) nach KVG und nach KVG, in v. Kaenel (Hg.), Krankentaggeldversicherung: Arbeits- und versicherungsrechtliche Aspekte, 2007, S. 78; Häberli, Sonderprobleme im Bereich des Arbeitsrechts, in v. Kaenel (Hg.), Krankentaggeldversicherung: Arbeits- und versicherungsrechtliche Aspekte, 2007, S. 150.
Gestützt auf Art. 98 Abs. 1 VVG handelt es sich bei Art. 87 VVG um eine einseitig zwingende Norm des VVG, das heißt, sie darf vertraglich nicht zu Ungunsten der Anspruchsberechtigten abgeändert werden ${ }^{84}$. Das Eidgenössische Versicherungsgericht hat Art. 87 VVG für die Taggeldversicherung nach KVG als analog anwendbar erklärt. Wenn dem Arbeitnehmer ein direktes Forderungsrecht gegenüber dem Versicherer zukommt, ist der Arbeitgeber von seiner Lohnfortzahlungspflicht befreit $^{85}$.

Der Zweck des selbstständigen Forderungsrechts besteht im Schutz der versicherten Arbeitnehmer ${ }^{86}$. Sie sollen davor geschützt sein, dass der Arbeitgeber die Zahlung der Versicherung entgegen nimmt und nicht an die versicherten Arbeitnehmer weiterleitet. Die Versicherung trägt das Risiko einer Doppelzahlung ${ }^{87}$. Die Leistung der Versicherung an den Versicherungsnehmer befreit die Versicherung nicht von der Leistung an die versicherte Person ${ }^{88}$. Das Obergericht des Kantons Zürich entschied mit Urteil vom 1. Juni 2001, eine Versicherung müsse eine bereits an den Arbeitgeber ausgerichtete, von diesem aber nicht weitergeleitete Leistung nochmals direkt an den versicherten Arbeitnehmer ausrichten. Der Arbeitgeber fiel in Konkurs, nachdem er die Zahlung der Versicherung entgegengenommen, aber noch nicht weitergeleitet hatte. Der Einwand der beklagten Versicherung, der versicherte Arbeitnehmer habe konkludent seine Zustimmung gegeben, dass die Versicherungsleistungen über seinen Arbeitgeber ausgerichtet werden, wurde vom Obergericht mit ausdrücklichem Verweis auf die einseitig zwingende Bestimmung in Art. 87 VVG nicht gehört $^{89}$.

\section{Steuerfolgen für Grenzgängerlnnen in Deutschland}

\section{Vorbemerkungen}

Bei Grenzgänger/innen stellt sich die Frage, wie die schweizerischen Leistungen bei krankheits- oder unfallbedingter Arbeitsunfähigkeit in Deutschland nach deutschem Steuerrecht

84 Stein in Honsell/Vogt/Schnyder, Basler Kommentar zum Bundesgesetz über den Versicherungsvertrag, 1. Auflage, 2001, N 16 zu Art. 87 VVG; Häberli, Sonderprobleme im Bereich des Arbeitsrechts, in v. Kaenel (Hg.), Krankentaggeldversicherung: Arbeits- und versicherungsrechtliche Aspekte, 2007, S. 150.

85 BGE 120 V 38, E. 3c/bb; BGE 122 V 81, E. 1b. Nach Egli, Lohnfortzahlung und Versicherungsschutz gemäß Art. 324a OR, AJP 2000, S. 1077, geht der Anspruch gegenüber dem Arbeitgeber trotz des direkten Forderungsrechts gegenüber der Kollektivversicherung nicht verloren.

86 Stein in Honsell / Vogt / Schnyder, Basler Kommentar zum Bundesgesetz über den Versicherungsvertrag, 1. Auflage, 2001, N 1 zu Art. 87 VVG.

87 Geiser, Fragen im Zusammenhang mit der Lohnfortzahlungspflicht bei Krankheit, in: AJP 2003 S. 332; Häberli, Sonderprobleme im Bereich des Arbeitsrechts, in v. Kaenel (Hg.), Krankentaggeldversicherung: Arbeits- und versicherungsrechtliche Aspekte, 2007, S. 150.

88 Geiser, Fragen im Zusammenhang mit der Lohnfortzahlungspflicht bei Krankheit, in AJP 2003, S. 332; Häberli, Sonderprobleme im Bereich des Arbeitsrechts, in v. Kaenel (Hg.), Krankentaggeldversicherung: Arbeits- und versicherungsrechtliche Aspekte, 2007, S. 150; Stein in Honsell / Vogt/ Schnyder (Hg.), Basler Kommentar zum Bundesgesetz über den Versicherungsvertrag, 1. Auflage, 2001, N 23 zu Art. 87 VVG.

89 Urteil des Obergerichts Zürich v. 1.6.2001, in plädoyer 6/2001, S. 86 ff. 
besteuert werden ${ }^{90}$. In Frage kommt nach 33 Ziff. 1 lit. a des Einkommensteuergesetztes (EStG) eine Steuerbefreiung: Leistungen der Krankenversicherung und Leistungen der Unfallversicherung werden nicht als Einkommen besteuert. Gleiches gilt nach §3 Ziff. 1 lit. d EStG für das Mutterschaftsgeld nach dem Mutterschutzgesetz. Arbeitslohn ist demgegenüber gestützt auf § 19 EStG zu versteuern. Zum Arbeitslohn gehören nach § 19 Abs. 1 Ziff. 1 Gehälter, Löhne, Gratifikationen, Tantiemen und andere Bezüge und Vorteile für eine Beschäftigung im öffentlichen oder privaten Dienst. Da die Arbeitgeber sich an den Prämien für die Kollektivkrankentaggeldversicherung beteiligen müssen ${ }^{91}$, ist $\mathrm{zu}$ prüfen, ob diese Beiträge steuerpflichtig sind.

\section{Beiträge des Arbeitgebers als steuerpflichtige Einkommen}

Nach § 2 Abs. 2 Ziff. 3 Satz 1 der Lohnsteuerdurchführungsverordnung (LStDV) gehören auch solche Ausgaben zum Arbeitslohn, die ein Arbeitgeber leistet, um Arbeitnehmer oder ihnen nahestehenden Personen für den Fall einer Krankheit, eines Unfalles, des Alters oder des Todes abzusichern ${ }^{92}$. Solche steuerpflichtigen Arbeitgeberbeiträge zur Zukunftssicherung der Arbeitnehmenden liegen dann vor, wenn die Arbeitnehmenden der Zukunftssicherung ausdrücklich oder stillschweigend zustimmen ${ }^{93}$ und wenn ihnen auf die Leistung ein unentziehbarer Rechtsanspruch zukommt ${ }^{94}$. Gestützt auf die ständige Rechtsprechung des Bundesfinanzhofes (BFH) finden diese Rechtsgrundsätze auch bei Grenzgängern Anwendung, deren schweizerische Arbeitgeber Beiträge an die Zukunftssicherung leisten $^{95}$.

Ausgangspunkt der Kollektivkrankentaggeldversicherung im Arbeitsverhältnis ist die vertragliche Regelung im Arbeitsvertrag ${ }^{96}$. Insoweit ist das Erfordernis der Zustimmung der Arbeitnehmenden zu den Beiträgen des Arbeitgebers an die Zukunftssicherung erfüllt. Wie weiter oben dargestellt wurde ${ }^{97}$, haben die in schweizerischen Kollektivkrankentaggeldversi-

90 Nach Art. 15a des Doppelbesteuerungsabkommens Schweiz - Deutschland (SR. 0.672.913.62, Abkommen zwischen der Schweizerischen Eidgenossenschaft und der Bundesrepublik Deutschland zur Vermeidung der Doppelbesteuerung auf dem Gebiete der Steuern vom Einkommen und vom Vermögen vom 11.8. 1971) erhebt die Schweiz bei Grenzgänger/innen eine Quellensteuer von 4,5\% auf den Bruttolohn. Zur Vermeidung der Doppelbesteuerung rechnet Deutschland die schweizerische Steuer auf seine eigene Steuer an. Siehe dazu: Baumer, Erwerbseinkünfte im internationalen Verhältnis, STR 2006, S. $330 \mathrm{ff}$., insbes. S. 342 .

91 Die Beteiligung der Arbeitgeber an den Prämien für die Kollektivkrankentaggeldversicherung ist zumindest in den Fällen erforderlich, in denen die Arbeitgeberin durch die Kollektivkrankentaggeldversicherung ihr Lohnfortzahlungsrisiko (oder einen Teil davon) abdeckt, siehe zum Ganzen weiter oben in diesem Text, II. 3. Leistungen durch die Kollektiv-Krankentaggeldversicherung, S. 12.

92 BFH-Urteil in STtBI II 2006, 528 zu II.4. Siehe dazu weiter: Thürmer, N 290, N 292 und N 294 zu § 19 EStG, in Blümich, Einkommensteuergesetz, Körperschaftsteuergesetz, Gewerbesteuergesetz, 96. Ergänzungslieferung, 2007.

93 Siehe $\$ 2$ Abs. 2 Ziff. 3 Satz 2 LStDV, weiter: BGH VI R 109/74 v. 15. 7. 1977, BStBI II 77, 761.

94 Siehe BFH, Urteil vom 12.4. 2007, VI R 55/05, BStBI. II 2006, 528.

$95 \mathrm{BFH}$, Entscheidungen vom 15. 11.2007, VI R 30/04, BFH/NV 2008, 550; vom 13.3. 2006, VI B 113/05, BFH/NV 2006, 1093; vom 13.4.2005, VI B 172/04.

96 Siehe dazu oben, II. 2. b) Abweichende Regelungen gemäß Art. 324a Abs. 4 OR.

97 Siehe dazu oben, II. 3. d) Direktes Forderungsrecht cherungen versicherten Arbeitnehmenden gegenüber der Versicherung ein direktes Forderungsrecht. Das direkte Forderungsrecht der versicherten Arbeitnehmenden kann nicht vertraglich abbedungen werden ${ }^{98}$. Damit sind die beiden Erfordernisse hinsichtlich der Qualifikation der Arbeitgeberbeiträge an die in der Schweiz nicht obligatorische Kollektivkrankentaggeldversicherung erfüllt und die Arbeitgeberbeiträge stellen Arbeitslohn dar. Anders verhält es sich mit den Arbeitgeberbeiträgen zu einer gesetzlich vorgesehenen Sozialversicherung. Solche Beiträge sind gestützt auf § 3 Ziff. 62 EStG steuerfrei ${ }^{99}$.

\section{Steuerpflicht der Lohnersatzleistungen}

Lohnfortzahlungen des Arbeitgebers bei Krankheit und Unfall fallen nicht unter den Befreiungstatbestand des §3 Ziff. 1 lit. a EStG und stellen steuerpflichtigen Arbeitslohn dar ${ }^{100}$. Steuerfrei sind demgegenüber gemäß diesem Paragraphen Leistungen aus einer Krankenversicherung, aus einer Pflegeversicherung und aus der gesetzlichen Unfallversicherung sowie nach §3 Ziff. 1 lit. d EStG das Mutterschaftsgeld nach dem Mutterschutzgesetz. Steuerfreie Einnahmen sind nach der Rechtsprechung Leistungen einer Krankentaggeldversicherung, die Versicherungsschutz gegen Verdienstausfall bietet ${ }^{101}$.

Diese - hier nur sehr gerafft dargestellte - Rechtslage nach deutschem Steuerrecht hat für die steuerliche Qualifikation schweizerischer Lohnersatzleistungen folgende Auswirkungen:

- Leistungen der obligatorischen Mutterschaftsversicherungen und der obligatorischen Unfallversicherung sind steuerfrei $^{102}$,

- Lohnfortzahlungsleistungen des Arbeitgebers nach öffentlichem Personalrecht oder nach Art. 324a Abs. 1-3 und nach Art. 324b OR stellen steuerpflichtigen Arbeitslohn dar.

Etwas komplexer ist die Rechtslage bezüglich der Leistungen aus einer Kollektivkrankentaggeldversicherung. Die Kollektivkrankentaggeldversicherung dient einerseits dem Arbeitgeber dazu, sein Risiko auf Lohnfortzahlung abzudecken (insbesondere bei langjährigen Mitarbeitenden, die längerdauernden gesetzlichen Anspruch auf Lohnfortzahlung haben) und andererseits gelangen die versicherten Arbeitnehmer durch die Kollektivkrankentaggeldversicherung in den Genuss eines ausreichenden Versicherungsschutzes gegen das Risiko des krankheitsbedingten Verdienstausfalles. Insoweit haben schweizerische Leistungen aus einer Kollektivkrankentaggeldversicherung

98 Die Bestimmung in Art. 87 VVG zum direkten Forderungsrecht ist eine zwingende Inhaltsbestimmung des Versicherungsvertrages.

99 Siehe dazu: Thürmer, N 280 und N 291 zu § 19 EStG, in Blümich, Einkommensteuergesetz, Körperschaftsteuergesetz, Gewerbesteuergesetz, 96. Ergänzungslieferung, 2007.

100 BFH, Urteil vom 4. 5. 2006, VI R 19/03, BStBI II 2006, 832; FG BaWü EFG 98, 718; Von Beckerrath, N 10 zu § 3 EStG, in Kirchhof, EStG, KompaktKommentar Einkommensteuergesetz, 6. neu bearbeitete Auflage, 2006; Drenseck, N 50 (Stichwort Lohnfortzahlung im Krankheitsfall) zu § 19 EStG, in Schmidt, Kommentar Einkommensteuergesetz, 26. Auflage 2007.

101 BFH BStBI II 69, 489; BEtBI II 83, 101 (104); Von Beckerrath, N 10 zu §3 EStG, in: Kirchhof, EStG, KompaktKommentar Einkommensteuergesetz, 6., neu bearbeitete Auflage, 2006.

102 Nach BFH, BStBI. II 96, 478 (480) sind auch Leistungen einer ausländischen gesetzlichen Unfallversicherung steuerbefreit. 
sowohl den Charakter eines Ersatzes einer Arbeitgeberlohnfortzahlung wie einer „reinen“ Versicherungsleistung. Entscheidend ist indes, dass den versicherten Arbeitnehmern in jedem Fall ein direktes Forderungsrecht gegenüber der Versicherung zukommt, was bestimmend für die Qualifikation der Arbeitgeberbeiträge an die Kollektivkrankentaggeldversicherung als steuerpflichtigen Arbeitslohn ist. Da die Arbeitgeberbeiträge bereits (steuerpflichtigen) Arbeitslohn darstellen, begründen die entsprechenden Versicherungsleistungen keinen weiteren Arbeitslohn ${ }^{103}$. Im Ergebnis fallen die schweizerischen Leistungen aus einer Kollektivkrankentaggeldversicherung unter den Befreiungstatbestand nach §3 Ziff. 1 lit. a EStG.

\section{Aspekte der Arbeitnehmerfreizügigkeit}

\section{Gleichbehandlungsansprüche nach dem FZA:}

\section{Auswirkungen auf Grenzgänger/innen}

Das FZA enthält in Art. 2 in Verbindung mit Art. 7 lit. a ein mit Art. 39 Abs. 2 EGV vergleichbares Diskriminierungsverbot aufgrund der Staatsangehörigkeit, wobei nicht der ausländische Arbeitnehmer an sich vor Diskriminierung geschützt ist, sondern vielmehr der europäische Wanderarbeitnehmer, der einen grenzüberschreitenden Sachverhalt verwirklicht hat ${ }^{104}$. Nach Art. 9 Abs. 2 des Anhang I des FZA haben europäische Wanderarbeitnehmende und ihre Familienangehörigen „die gleichen steuerlichen und sozialen Vergünstigungen wie die inländischen Arbeitnehmer und ihre Familienangehörigen“. Diese Bestimmung ist Art. 7 Abs. 2 der V0 1612/68/EWG nachgebildet. Anspruch auf Gleichbehandlung besteht gestützt auf Art. 9 Abs. 1 und Abs. 4 Anhang I FZA auch hinsichtlich Beschäftigungsbedingungen entlang sämtlicher Phasen eines Arbeitsverhältnisses. Auch diese Regelung entspricht derjenigen in der V0 1612/68/EWG. Hinzuweisen ist auf Art. 16 Abs. 2 FZA. Diese Bestimmung verpflichtet die Vertragspartien zur Berücksichtigung der EuGH-Rechtsprechung zur Arbeitnehmerfreizügigkeit. Gemäss Art. 16 Abs. 2 FZA ist diese Berücksichtigungspflicht indes auf den Zeitpunkt bis zur Unterzeichnung des Abkommens (21. Juni 1999) beschränkt. Nach der Lehre und bisheriger Praxis ist jedoch davon auszugehen, dass diese beschränkte Berücksichtigungspflicht für lediglich präzisierende EuGH-Rechtsprechung nicht gilt ${ }^{105}$. Somit ist die EuGH-Rechtsprechung zum Diskriminierungs- und Beschränkungsverbot gegenüber europäischen Wanderarbeitnehmenden im Anwendungsbereich des FZA weitestgehend anwendbar. Im vorlie-

103 BFH-Beschluss vom 23.7. 1999, VI B 166/99, STtBI II 1999, 684, zu 2.a. Nicht relevant ist, ob der Arbeitgeber die Versicherungsleistung entgegennimmt und an den Arbeitnehmer weiterleitet, siehe BFH-Beschluss VI B $172 / 04$.

104 EuGH v. 6. 6. 2000 - Rs. C-281/98, Rz 41, Angonese ./. Casse die Risparmio di Bolzano SpA. Siehe zur Analogie dieser Rechtsprechung nach dem FZA: Hangartner, Der Grundsatz der Nichtdiskriminierung wegen der Staatsangehörigkeit im Freizügigkeitsabkommen der Schweiz mit der Europäischen Gemeinschaft, AJP 2003, S. 263; Pärli, Arbeitsrechtliches Diskriminierungsverbot europäischer Wanderarbeitnehmer nach Gemeinschaftsrecht und nach Personenfreizügigkeitsrecht mit der Schweiz, ZESAR 2007, S. 33.

105 Siehe dazu ausführich: Imhof, Das Freizügigkeitsabkommen EG-Schweiz und seine Auslegungsmethode, ZESAR 2007, S. 155 ff., ZESAR 2007, S. 217 ff. genden Zusammenhang trifft dies namentlich auf die EuGHRechtsprechung zum Anspruch auf gleiche steuerliche Vergünstigungen $\mathrm{zu}^{106}$.

Angehörige der EU-Mitgliedstaaten und Schweizerinnen und Schweizer können sich in jedem EU-Staat auf die unmittelbar anwendbaren Bestimmungen des FZA inkl. Anhang als Bestandteil des Gemeinschaftsrechts berufen. Gerichte der Mitgliedstaaten sind berechtigt bzw. verpflichtet, Auslegungsfragen des FZA dem EuGH zu Vorabentscheidung vorzulegen $^{107}$. Anders ist die Rechtslage in der Schweiz. Hier können die unmittelbar anwendbaren Bestimmungen des FZA zwar vor jedem schweizerischen Gericht angerufen werden; das schweizerische Bundesgericht ist indes für die Auslegung des FZA in letzter Instanz zuständig ${ }^{108}$.

Die in diesem Beitrag aufgezeigte Rechtslage bezüglich schweizerischer Leistungen bei Arbeitsunfähigkeit ist hinsichtlich der Gleichbehandlungsansprüche nach FZA und Anhang in verschiedener Hinsicht von Bedeutung. Zum einen fallen Lohnfortzahlungsleistungen des Arbeitgebers in den Anwendungsbereich der V0 1612/68/EG ${ }^{109}$. Angesichts der arbeitsvertraglichen Grundlage gehören auch Leistungen aus der Kollektivkrankentaggeldversicherung zu Leistungen, die den europäischen Wanderarbeitnehmenden diskriminierungsfrei zu gewähren sind. Zum anderen sind allfällige steuerliche Benachteiligungen als Folge der Verwirklichung eines grenzüberschreitenden Sachverhaltes im Lichte des Gleichbehandlungsanspruches zu würdigen.

Angesichts der Verschiedenheit der sozial- und steuerrechtlichen Systeme der EU-Staaten und vorliegend den FZA-Vertragsstaaten ist die richtige dogmatische Erfassung eines Diskriminierungstatbestandes besonders anspruchsvoll ${ }^{110}$. Aus diesem Grund wird nachfolgend kurz die EuGH-Rechtsprechung zum Anspruch des europäischen Wanderarbeitnehmers auf gleiche steuerliche Vergünstigungen dargestellt und es wird - ausgehend von der Anwendbarkeit dieser Rechtspre-

106 Siehe dazu sogleich IV. 2. EuGH-Rechtsprechung zu steuerlichen Vergünstigungen und das FZA, S. 22.

107 Der EuGH ist gemäß Art. 220 EGV für die Wahrung des Rechts zuständig, dazu gehören auch die Abkommen mit Drittstaaten. Siehe dazu: EuGH v. 6.6. 2006 - Rs. C-339/05, Schlussanträge des Generalanwalts, Zentralbetriebsrat der Landeskrankenhäuser Tirols ./. Land Tirol (Das Vorabentscheidungsgesuch wurde zurückgezogen), Rz. 29, mit Hinweisen auf EuGH v. 30.9. 1987 - Rs. 12/86, Demirel./.Stadt Schwäbisch Gmünd. Siehe weiter: Pärli, Arbeitsrechtliches Diskriminierungsverbot europäischer Wanderarbeitnehmer nach Gemeinschaftsrecht und nach Personenfreizügigkeitsrecht mit der Schweiz, ZESAR 2007, S. 31.

108 Imhof, Das Freizügigkeitsabkommen EG-Schweiz und seine Auslegungsmethode, ZESAR 2007, S. 155 ff., insbes. S. 159 und die Hinweise in Fn. 36 und 37.

109 Nach der Rechtsprechung des EuGH fallen sie gleichzeitig unter die V0 1408/71/EWG, siehe EuGH v. 3.6.1992 - Rs. C-45/90, Paletta ./. Brennet, Rz 17 und neuerdings EuGH v. 18.1.2007 - Rs. C-332/05, Aldo Celozzi ./. Innungskrankenkasse Baden-Württemberg, Rz 20. Siehe zu dieser Entscheidung die Anmerkungen von Eichenhofer in ZESAR 2007, S. $237 \mathrm{ff}$.

110 Die Komplexität wird noch etwas erhöht, wenn in einem Sachverhalt nicht nur die jeweils nationalen Steuer- und Sozialrechtssystem und die gemeinschaftlichen Regelungen zur Arbeitnehmerfreizügigkeit und dem koordinierenden europäischen Sozialrecht anwendbar sind sondern darüber hinaus noch internationale Steuerabkommen zu berücksichtigen sind, siehe dazu EuGH v. 18. 1.2007 - Rs. C-332/05, Aldo Celozzi ./. Innungskrankenkasse Baden-Württemberg, und die Schlussbemerkung von Eichenhofer in der Urteilsbesprechung in ZESAR 2007, S. 241. 
chung auf das FZA - geprüft, welche Konsequenzen sich daraus für die steuerliche Behandlung der schweizerischen Ersatzeinkommen für deutsche Grenzgänger/innen ergeben.

\section{EuGH-Rechtsprechung zu steuerlichen Vergünstigungen und das FZA}

\section{a) Rechtsprechungsüberblick}

Direkte Steuern fallen in die Zuständigkeit der Mitgliedstaaten, die diese Befugnisse jedoch unter Wahrung des Gemeinschaftsrechts auszuüben und jede unmittelbare und mittelbare Diskriminierung aufgrund der Staatsagehörigkeit zu unterlassen haben $^{111}$. Die Mitgliedstaaten dürfen die Arbeitnehmerfreizügigkeit durch die Ausgestaltung des Steuersystems auch nicht ohne Rechtfertigungsgründe beschränken ${ }^{112}$. Der Gerichtshof hat wiederholt die Kohärenz der nationalen Steuersysteme als Rechtfertigungsgründe zugelassen. Voraussetzung ist indes ein direkter Zusammenhang zwischen einer steuerlichen Vergünstigung, z.B. der Abzugsfähigkeit von Beiträgen an die Altersund Todesfallversicherung, und der Besteuerung der zur Durchführung dieser Verträge erhaltener Beiträge ${ }^{113}$. Nicht mehr mit dem Kohärenzargument gerechtfertigt werden kann eine nationale Regelung, die dazu führt, dass ein Steuerpflichtiger bei der Berechnung seiner Einkommensteuer im Wohnsitzstaat einen Teil des Steuerfreibetrages und der persönlichen Steuervorteile verliert, weil im gleichen Bemessungsjahr noch in einem anderen Mitgliedstaat Einkünfte erzielt worden sind, die dort ohne Berücksichtigung der persönlichen und familiären Situation besteuert worden sind ${ }^{114}$. Unzulässig ist weiter, wenn mit steuerrechtlichen Maßnahmen bezweckt wird, den Nichtanschluss an ein System der sozialen Sicherheit auszugleichen ${ }^{115}$, wenn für die Anwendung eines für verheiratete Steuerpflichtige günstigeren Splittingtarifs ein inländischer Wohnsitz beider Ehegatten verlangt wird ${ }^{116}$ oder Beiträge an die Sozialversicherung nicht steuerlich von den Erwerbseinkünften abgezogen werden, falls diese Versicherungsträger ihren Sitz im Ausland haben und vom fraglichen Mitgliedstaat nicht als gleichwertig anerkannt sind ${ }^{117}$. Eine Beeinträchtigung der Arbeitnehmerfreizügigkeit stellen nationale Regelungen dar, die Steuererleichterungen nur für Beiträge in inländische Pensionsfonds gewähren $^{118}$.

111 EuGH v. 11.8.1995 - Rs. C-80/94, Wielockx ./. Inspecteur der directe belastingen, Rz 16; EuGH v. 13.12. 1994 - Rs. C-279/93, Finanzamt Köln-Altstadt./.Roland Schumacker, Rz. 21 und Rz. 26; EuGH v. 27.6. 1996 Rs. C-107/94, Asscher./.Staatssecretaris van Financiën, Rz 36.

112 EuGH v. 12.12.2002 - Rs. C-385/02, F.W.L. de Groot ./. Staatssecretaris van Financiën, Rz. 81- Rz. 95 (prüfen der Vorliegen einer Beschränkung), Rz. 96110 (prüfen des Vorliegens von die Beschränkung verneinenden Rechtfertigungsgründen).

113 EuGH v. 28. 1. 1992 - Rs. C-204/90, Bachmann ./. Belgien, Rz. 28; EuGH v. 28.1. 1992 - Rs. C-300/90, Kommission ./. Belgien, Rz. 21.

114 EuGH v. 12.12.2002 - Rs. C-385/02, F.W.L. de Groot ./. Staatssecretaris van Financiën, Rz. 110.

115 EuGH v. 27.6. 1996 - Rs. C-107/94, P.H. Asscher ./. Staatssecretaris van Financiën, Rz. 61.

116 EuGH v. 16. 5. 2000 - Rs. C-87/99, Patrick Zurstrassen ./. Administration des contributions directes, Rz 20.

117 EuGH v. 28. 1. 1992 - Rs. C-204/90, Bachmann ./. Belgien, Rz. 9 ff.; EuGH v. 28.1. 1992 - Rs. C-300/90, Kommission ./. Belgien, Rz. $7 \mathrm{ff}$.

118 EuGH v. 30. 1.2007 - Rs. C-150/04, Kommission ./. Dänemark, Rz. 77.

\section{b) Bedeutung für die Besteuerung von Grenzgänger/innen}

Der Zweck des gemeinschaftsrechtlichen Anspruchs auf gleiche steuerliche Vergünstigungen für europäische Wanderarbeitnehmende besteht darin, zusammen mit den anderen Bestimmungen über die Freizügigkeit zu verhindern, dass Gemeinschaftsangehörige benachteiligt werden, wenn sie im Gebiet eines anderen Mitgliedstaates eine wirtschaftliche Tätigkeit ausüben ${ }^{119}$. Diese Rechtslage findet gestützt auf das FZA auf Sachverhalte von Grenzgänger/innen Anwendung, die in Deutschland wohnen und in der Schweiz arbeiten. Die Arbeitnehmereigenschaft ist auch dann erfüllt, wenn die Grenzgänger/innen wie vorliegend Lohnersatzleistungen aus dem Arbeitsverhältnis beziehen. In Frage stehen die steuerlichen Folgen einer als Arbeitnehmende in FZA - Vertragsstaaten ausgeübten Tätigkeit ${ }^{120}$. Die deutschen Grenzgänger/innen in der Schweiz haben demzufolge gestützt auf das FZA grundsätzlich Anspruch darauf, dass sie bei der Besteuerung ihres Einkommens im Vergleich zu den rein inländischen Beschäftigten nicht unmittelbar oder mittelbar diskriminiert werden. Diese Grundsätze gelten auch bei der deutschen Besteuerung von Grenzgänger/innen, die schweizerische Lohnersatzleistungen beziehen. In diesem Beitrag wurde aufgezeigt, wie eine für Grenzgänger/innen nachteilsfreie Besteuerung möglich ist.

Komplexer ist die Situation hinsichtlich der Krankenpflegeversicherung. In Deutschland erhalten freiwillig in der gesetzlichen Krankenversicherung versicherte Arbeitnehmer gemäß § 3 Nr. 62 EStG jenen Teil des Arbeitgeberbeitrages steuerbefreit, welchen der Arbeitgeber aufgrund sozialversicherungsrechtlichen oder anderen gesetzlichen Vorschriften zu leisten verpflichtet ist. In der Schweiz krankenversicherte Grenzgänger/innen haben keinen gesetzlichen Anspruch auf Arbeitgeberbeiträge und die voll von den Grenzgänger/innen zu tragenden Krankenversicherungsprämien wirken sich in Deutschland außer im beschränkten Rahmen des §10 EStG nicht steuerbefreiend aus. Vergeblich machte ein in der Schweiz beschäftigter Grenzgänger vor dem Finanzamt und Finanzgericht geltend, ihm seien 50 Prozent seines Beitrages zu Krankenversicherung als fiktiver Arbeitgeberbeitrag steuerfrei zu erlassen. Der Bundesfinanzhof sieht darin keinen Verstoß gegen das verfassungsrechtliche Gleichheitsgebot nach Art. 3 Abs. 3 des Grundgesetzes; §3 Nr. 62 EStG unterscheide nicht zwischen Grenzgänger/innen und in Deutschland beschäftigten Arbeitnehmer/innen, vielmehr werde zwischen gesetzlich verpflichteten und gesetzlich nicht verpflichteten Arbeitgebern (bzw. deren Beiträgen) unterschieden ${ }^{121}$. Es fragt sich indes, ob die fehlende Abzugsfähigkeit der Krankenversicherungsbeiträge im Ergebnis nicht einer nach FZA verbotenen Behinde-

\footnotetext{
119 Schlussanträge vom 20.6.2002 des Generalanwaltes Philippe Léger in der Rs C-385/00, F.W.L. de Groot ./. Staatsecretaris van Fianciën, Rz. 25 mit Hinweisen auf die EuGH-Urteile v. 7.7.1988 - Rs. 143/87, Stanton ./. Inasti, Rz. 13 und v. 15.12. 1995 - Rs. C-415/93, Union royale belge des sociétés de football association and others ./. Bosman and others, Rz. 94.

120 EuGH v. 12.12.2002 - Rs. C-385/00, F.W.L. de Groot./. Staatssecretaris von Financiën, Rz. 82

121 BFH, Urteil vom 18. 12.2007, VI R 13/05, Erw. 2a/cc.
} 
rung der Arbeitnehmerfreizügigkeit gleichkommt. In Deutschland steuerpflichtige Grenzgänger/innen können die von ihnen selbst zu tragenden Krankenversicherungsbeiträge weder in der Schweiz noch in Deutschland von der Steuer absetzen, während dies wie erwähnt in Deutschland Beschäftigten zur Hälfte (der vom Arbeitgeber zu leistenden Prämien) möglich ist. Soweit ersichtlich musste sich der EuGH bis heute noch nie mit einer solchen Konstellationen auseinandersetzen. Im Lichte der bisherigen Entscheidungen zu „gleichen steuerlichen Vergünstigungen“ ist nicht auszuschließen, dass eine entsprechende Vorabentscheidungsanfrage zu Gunsten der Grenzgänger/innen beantwortet würde. 